\title{
Oxidative Stress Markers in Tubal Ectopic Pregnancy
}

\section{(1) Seyhun SUCU, ${ }^{a}$ \\ (1) M. Hanifi BADEMKIRAN, \\ D Hüseyin Çăglayan ÖZCAN, ${ }^{\mathrm{a}}$ \\ (1) Özge KÖMÜRCÜ, ${ }^{a}$ \\ D Denizhan BAYRAMOĞLU, ${ }^{\circ}$ \\ (1) Özcan BALATa}

aDepartment of Obstetrics and Gynecology, Gaziantep University Faculty of Medicine, Gaziantep

${ }^{b}$ Department of Obstetrics and Gynecology, Universtity of Health Sciences

Gazi Yaşargil Training and

Research Hospital,

Diyarbakır

'Clinic of Obstetrics and Gynecology,

Cengiz Gökçek Obstetrics and

Childeren'sHospital,

Gaziantep

Received: 31.12.2017

Received in revised form: 05.03.2018

Accepted: 19.03.2018

Available online: 10.04 .2018

Correspondence:

M. Hanifi BADEMKIRAN

Universtity of Health Sciences

Gazi Yaşargil Training and

Research Hospital,

Department of Obstetrics and Gynecology,

Diyarbakır, TURKEY

hanifibademkiran@hotmail.com

\begin{abstract}
Objective: In this study, a novel automated method was used to evaluate the levels of oxidative stress markers in patients with ectopic tubal pregnancy. Material and Methods: This prospective study investigated 30 patients diagnosed with tubal ectopic pregnancy (patient group), 30 healthy pregnant women in their first trimester (first control group), and 30 healthy women who were not pregnant (second control group). All the participants were admitted to the Department of Obstetrics and Gynecology, Gaziantep University Medical School, Turkey. The Erel method was used to measure the total oxidant level (TOL), total antioxidant capacity (TAC), and oxidative stress index (OSI). Results: TOL and OSI values were significantly higher in the patient group ( $\mathrm{p}=$ 0.001 ) than in the second control group, while there was no significant difference between the patient group and the first control group. The values for TAC were significantly lower in the patient group ( $\mathrm{p}=0.001$ ) compared to the second control group; however, there was no significant difference between the patient group and the first control group $(\mathrm{p}=0.449)$. Conclusion: The role of oxidative stress in the etiopathogenesis of tubal ectopic pregnancy warrants more comprehensive studies in the future.
\end{abstract}

Keywords: Ectopic pregnancy; total oxidant level; total antioxidant capacity; oxidative stress index

he implantation of the fertilized ovum in a region other than the uterine cavity is known as ectopic pregnancy. It is a complication observed in $1 \%-2 \%$ of all pregnancies. ${ }^{1}$ Despite the progress in diagnostic techniques, ectopic pregnancy remains a crucial cause of maternal morbidity and mortality throughout the world, especially in the countries where prenatal care is poor. ${ }^{2}$ More than $95 \%$ of the ectopic pregnancies are located in the tuba. ${ }^{3}$ The etiopathogenesis of tubal ectopic pregnancy has not been clarified yet, though this condition is usually associated with tubal abnormalities and dysfunction, such as abnormal tubal-cilia activity or contractility. ${ }^{4}$ Reactive oxygen species produced during metabolic and physiological processes occurring in the body are known as oxidants. Antioxidants play a role in reducing the harmful effects that the oxidants cause in the body. There is a delicate balance between oxidants and antioxidants inside the cells, and if this balance tips in favor of oxidants, a condition of oxidative stress occurs, which may cause cellular damage. ${ }^{5}$ Any damage that occurs in the tubas at a cellular level may predispose women to a defective tubal transport of the embryo, which is a central process in ectopic preg- 
nancy. The aim of this prospective study was to measure and compare the levels of oxidative stress markers in tubal ectopic pregnancy using a novel automated method developed by Erel.,

\section{STATISTICAL ANALYSIS}

All data were analyzed using SPSS version 11.5 for Windows. Mann-Whitney U test was used to compare two independent groups with no normal distribution. Comparisons among more than two groups were performed by using ANOVA when the variables were normally distributed, and by using the Kruskal-Wallis test when the variables were non-normally distributed. Dunn's multiple comparisons test was used to determine the significant subgroups. The statistical significance threshold was set at $\mathrm{p}<0.05$.

\section{MATERIAL AND METHODS}

This prospective study was performed at the University Hospital for a period of one year. This study included 30 patients with tubal ectopic pregnancy (patient group), 30 healthy pregnant women in their first trimester (first control group), and 30 healthy women who were not pregnant and without any medical problems (second control group). Informed consent was obtained from all the participants prior to the study. Approval was obtained from the institutional review board. Transvaginal ultrasound and serial measurements of serum betahuman chorionic gonadotropin $(\beta-\mathrm{Hcg}$ ) levels were used to diagnose the ectopic pregnancy. The diagnostic criteria for ectopic pregnancy were an irregular increase in the $\beta$-Hcg levels, ectopic tubal implantation of pregnancy (gestational sac containing yolk sac and/or fetal heartbeat) observed in the transvaginal ultrasound, or the presence of pathological diagnosis. Ectopic pregnancies were managed medically and/or surgically. Participants who smoked, consumed alcohol, or used antioxidants or drugs were excluded from this study. Those who were diagnosed with any systemic disease were also excluded.

Patient and control groups were asked to abstain from taking any medication on the night before the study. On the morning of the study, $8 \mathrm{cc}$ blood samples were taken from all the participants. The samples were centrifuged at $1500 \times \mathrm{g}$ for $10 \mathrm{~min}$ to obtain sera. The sera were placed in Eppendorf tubes for one min and stored at $-80{ }^{\circ} \mathrm{C}$ until the TAC and TOL evaluation. A fully automated method developed by Erel was used to evaluate TOL and TAC. ${ }^{6,7}$ In this novel automated method, antioxidants bleach the characteristic color of a more stable radical cation, 2,2' -azino-bis (3-ethylbenzothiazoline-6-sulfonic acid) (ABTS). The precision value of the assay was $3 \%$. The results were expressed as mmol Trolox equivalent/L. The ferrous ion, o-dianisidine complex, was oxidized to ferric ion by the oxidants present in the sample. The abundance of glycerol molecules in the reaction medium led to the enhancement of the oxidation reaction. A complex was formed by the ferric ion with Xylenol orange in the acidic medium. There was a significant relationship between the spectrophotometrically measured color intensity and the total amount of oxidant molecules in the sample. The assay was calibrated using hydrogen peroxide and the results were expressed in terms of $\mu \mathrm{mol} \mathrm{H}_{2} \mathrm{O}_{2}$ equivalent/L. The oxidative stress index (OSI) was calculated as the ratio of TOL to TAC. In order to complete this calculation, TAC results were converted to $\mathrm{mmol} / \mathrm{L}$, and the following formula was used to find the OSI value: OSI (arbitrary unit)=TOL $\left(\mu \mathrm{mol} \mathrm{H}_{2} \mathrm{O}_{2}\right.$ equivalent/L)/TAC (mmol Trolox equivalent/L). ${ }^{8}$

TABLE 1: Comparison of demographic and obstetric parameters in the patient and control groups.

\begin{tabular}{|lcccc|}
\hline Variable & Patient $(\mathbf{n}=\mathbf{3 0})$ & First Control Group $(\mathbf{n}=\mathbf{3 0})$ & Second Control Group $(\mathbf{n}=\mathbf{3 0})$ & $\mathbf{p}$ \\
\hline Age & $28.3 \pm 5.65$ & $27.60 \pm 6.75$ & $27.93 \pm 6.87$ & 0.930 \\
\hline Body mass index $\left(\mathrm{kg} / \mathrm{m}^{2}\right)$ & $26.05 \pm 3.10$ & $26.92 \pm 6.11$ & $26.55 \pm 4.74$ & 0.782 \\
\hline Parity & $0.96 \pm 1.12$ & $1.60 \pm 1.52$ & $1.06 \pm 1.25$ & 0.226 \\
\hline Gestational age & $5.60 \pm 1.67$ & $6.02 \pm 2.13$ & - & 0.398 \\
\hline
\end{tabular}


TABLE 2: Total antioxidant capacity (TAC), total oxidant level (TOL), and oxidative stress index (OSI) results for the patient and control groups.

\begin{tabular}{|lcccc|}
\hline Variable & Patient $(\mathbf{n}=\mathbf{3 0})$ & First Control Group ( $\mathbf{n = 3 0})$ & Second Control Group (n=30) & $\mathbf{p}$ \\
\hline Total antioxidant capacity (mmolTrolox Equiv./L) & $0.98 \pm 0.16^{\dagger}$ & $1.04 \pm 0.13^{\ddagger}$ & $1.25 \pm 0.21$ & 0.001 \\
Total oxidant level ( $\mu$ mol H2O2 Equiv./L) & $14.44 \pm 16.07^{\dagger}$ & $8.76 \pm 7.05^{\ddagger}$ & $3.62 \pm 1.43$ & 0.001 \\
\hline Oxidative stress index (arbitrary unit) & $1.39 \pm 1.57^{\dagger}$ & $0.84 \pm 0.65^{\ddagger}$ & $0.29 \pm 0.12$ & 0.001 \\
\hline
\end{tabular}

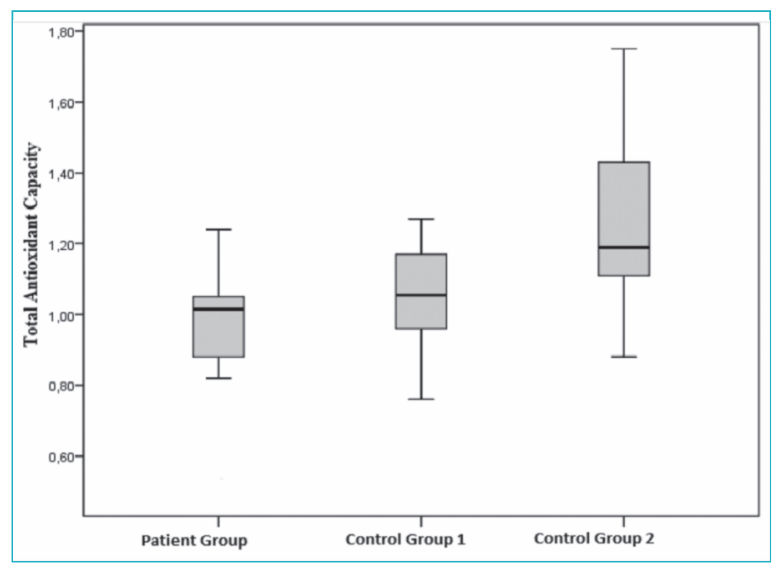

FIGURE 1: Total antioxidant capacity (TAC) for all groups (mmolTroloxEquiv./L).

\section{RESULTS}

The patient and control groups did not vary significantly in terms of demographic and clinical data (Table 1). Table 2 enlists the TAC, TOL, and OSI values for the patient and control groups. TAC was significantly lower in the patient group compared to second control group $(\mathrm{p}=0.001)$; however, there was no statistically significant difference in TAC between the patient group and the first control group $(\mathrm{p}=0.449)$ (Figure 1$)$. When compared to the second control group, the patient group exhibited significantly higher TOL and OSI ( $\mathrm{p}=0.001)$; however, there was no statistically significant difference between the patient group and the first control group ( $\mathrm{p}=0.661, \mathrm{p}=0.919)$ (Figure 2 and 3).

\section{DISCUSSION}

Lately, the diagnosis of ectopic pregnancy has improved due to the advances in diagnostic methods,

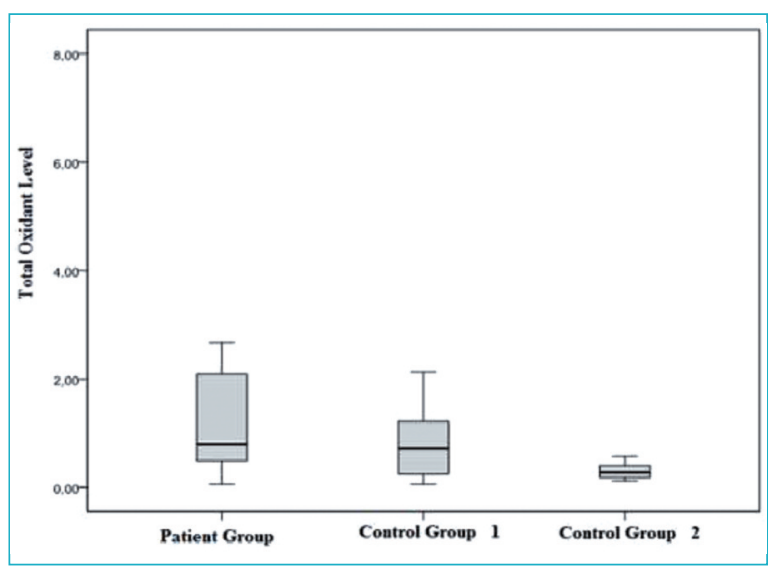

FIGURE 2: Total oxidant level (TOL) for all groups ( $\mu \mathrm{mol} \mathrm{H} 2 \mathrm{O} 2$ Equiv./L).

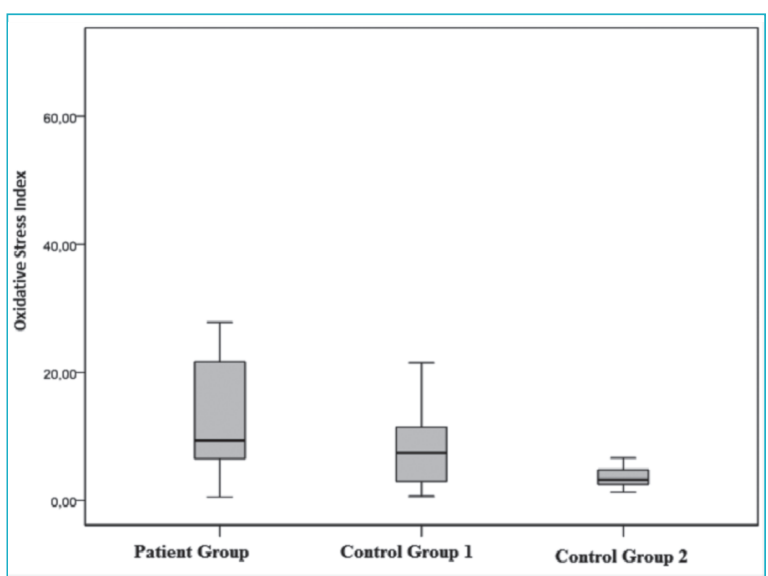

FIGURE 3: Oxidative stress index (OSI) for all groups (arbitrary unit).

increased risk factors, and increased use of assisted reproduction. ${ }^{9}$ It is crucial to diagnose an ectopic pregnancy early, because a delay in the diagnosis may lead to the hemorrhagic maternal death and future infertility. ${ }^{10}$ The primary aim of the modern management of ectopic pregnancy, in addition to 
saving lives, is to preserve fertility. The most important cause of maternal mortality in the first trimester is an ectopic pregnancy, and it accounts for about $5 \%-6 \%$ of all maternal deaths in the United States. ${ }^{11}$ The aim of this study was to investigate whether the oxidative stress levels were high enough to act as a possible etiological cause in patients with ectopic pregnancy. Oxidative stress plays an important role in the pathophysiology of several diseases, including pregnancy complications. ${ }^{12}$ The balance between oxygen radical formation and the endogenous antioxidant capacity plays a crucial role in the damage caused in tissues by oxidative stress. ${ }^{13}$ The development of an embryo before it is properly implanted may be affected by an imbalance between the production of toxic compounds, such as oxygen-based free radicals and lipid peroxides, and the detoxification and scavenging of these harmful molecules in vivo. ${ }^{14}$ Moreover, the pathological generation of nitric oxide synthesized from L-arginine in different tissues may reduce tubal cilia movements and smooth muscle contractions. Increased synthesis of nitric oxide isoforms has an impact on embryo transport, leading to the possibility of tubal ectopic pregnancy. ${ }^{4}$ Therefore, oxidative stress may disturb the tubal microenvironment and lead to ectopic pregnancy.

Researchers are searching for novel markers to reduce the time it takes the clinicians to diagnose an ectopic pregnancy, in order to reduce the likelihood of tubal rupture. The previous studies have investigated the use of placental pregnancy-associated plasma protein A, human placental lactogen, inhibin $A$, activin $A$, and non-placental markers (glycodelin and vascular endothelial growth factor) to diagnose ectopic pregnancy. ${ }^{15,16}$ Our study is the second one to investigate oxidative stress in ectopic pregnancy. Hilali and colleagues investigated the oxidative status and serum prolidase activity in tubal ectopic pregnancy. ${ }^{17}$ Although the present study supported the conclusions of the study by Hilali et al., the differences between the women with ectopic pregnancy and the healthy pregnant women in their first trimester were not statistically significant in our study. In order to assess the difference between intrauterine and ectopic pregnan- cies, we included two control groups in our study, which consisted of healthy pregnant women in their first trimester and healthy women who were not pregnant. In both the control groups, TAC levels were higher than those in the patient group, though the difference was not significant between the patient group and the first control group. In the healthy pregnant women in their first trimester, TOL and OSI values were lower than those in the patient group, though this difference was also not statistically significant.

The association between smoking and increased rate of ectopic pregnancy was demonstrated by Shao et al. in a retrospective case-control study. ${ }^{18}$ Human and animal experiments have demonstrated that the fallopian tube tissue was damaged and underwent functional loss as a result of the effects of harmful substances produced during smoking, and therefore, this condition might predispose women to ectopic pregnancy. Smoking was an exclusion criterion in the present study. However, endogenous oxidants of a nature similar to those associated with smoking may share a common pathogenetic pathway in ectopic pregnancies, causing functional damage to the tubas. The oxidant and antioxidant level measurement methods used in contemporary research are based on chemiluminescence, fluorescence, or calorimetric methods. ${ }^{19,20}$ However, these measurement methods are not cost-effective, and a longer time is needed to carry out the study. In contrast, the present study used a more cost-effective and reliable measurement method, a novel, fully automated measurement method developed by Erel. ${ }^{21}$ Although the sample size of the present study does not allow for conclusive statements to be made, higher TOL and OSI values and lower TAC values were observed among the women with tubal ectopic pregnancy, compared to healthy women without pregnancy. We hypothesize that an increase in TOL and OSI may contribute to the pathogenesis of tubal ectopic pregnancy.

\section{CONCLUSION}

Elevated TOL and OSI in the patients with ectopic pregnancy compared to the non-pregnant healthy 
controls pave the way for future studies regarding the pathogenesis, diagnosis, and/or early treatment of ectopic pregnancy. However, the differences in these markers between the normal early pregnancy and the ectopic pregnancy were not significant, which could be attributed to the small sample size used in this study. Large-scale laboratory studies are therefore required in order to understand the correlation between these markers and ectopic pregnancy.

\section{Source of Finance}

During this study, no financial or spiritual support was received neither from any pharmaceutical company that has a direct connection with the research subject, nor from a company that provides or produces medical instruments and materials which may negatively affect the evaluation process of this study.

\section{Conflict of Interest}

No conflicts of interest between the authors and / or family members of the scientific and medical committee members or members of the potential conflicts of interest, counseling, expertise, working conditions, share holding and similar situations in any firm.

\section{Authorship Contributions}

Idea/Concept: Özcan Balat, Seyhun Sucu; Design: H. Çağlayan Özcan; Control/Supervision: Seyhun Sucu; Data Collection and/or Processing: Özge Kömürcü, Denizhan Bayramoğlu; Analysis and/or Interpretation: Seyhun Sucu, M. Hanifi Bademkıran; Literature Review: M. Hanifi Bademkıran; Writing the Article: Seyhun Sucu, M. Hanifi Bademkıran; Critical Review: Özcan Balat, Seyhun Sucu, H.Çağlayan Özcan; Materials: Özge Kömürcü, Denizhan Bayramoğlu.
1. Farquar MC. Ectopic pregnancy. Lancet 2005;366(9485):583-91.

2. Paxton A, Maine D, Freedman L, Fry D, Lobis S. The evidence for emergency obstetric care. Int J Gynecol Obstet 2005;88(2):181-93.

3. Stovall TG, McCord ML. Early pregnancy loss and ectopic pregnancy. In: Berek JS, Adashi EY, Hillard PA, eds. Novak's Gynecology. 12th ed. Baltimore: Maylar Williams \& Wilkins; 1996. p.423-87.

4. Shao R, Zhang SX, Weijdegård B, Zou S, Egecioglu $E$, Norström $A$, et al. Nitric oxide synthases and tubal ectopic pregnancies induced by Chlamydia infection: basic and clinical insights. Mol Hum Reprod 2010;16(12):907-15.

5. Halliwell B, Gutteridge JM. Lipid peroxidation, oxygen radicals, cell damage, and antioxidant therapy. Lancet 1984;1(8391):1396-7.

6. Erel O. A novel automated method to measure total antioxidant response against potent free radical reactions. Clin Biochem 2004;37(2):112-9.

7. Erel O. A new automated colorimetric method for measuring total oxidant status. J Clin Biochem 2005;38(12):1103-11.

8. Ozturk E, Balat O, Acılmıs YG, Ozcan C, Pence S, Erel Ö. Measurement of the placen-

\section{REFERENCES}

tal total antioxidant status in preeclamptic women using a novel automated method. J Obstet Gynaecol Res 2011;37(4):337-42.

9. Nguyen PKP, Wallach EE. Contemporary diagnosis and management ofectopic pregnancy. Clin Obstet Gynecol 1997;17:1-5.

10. Rajendiren S, Dhiman P. Biomarkers of ectopic pregnancy-present and future. contemporary gynecologic practice. Intech 2015;2(51).

11. Anderson FW, Hogan JG, Ansbacher R. Sudden death: ectopic pregnancy mortality. Obstet Gynecol 2004;103(6):1218-23.

12. Al-Gubory KH, Fowler PA, Garrel C. The roles of cellular reactive oxygen species, oxidative stress and antioxidants in pregnancy outcomes. Int $\mathrm{J}$ Biochem Cell Biol 2010;42(10):1634-50.

13. Burton GJ, Jauniaux E. Oxidative stress. Best Pract Res Clin Obstet Gynaecol 2011;25(3):287-99.

14. Lopes AS, Lane M, Thompson JG. Oxygen consumption and ROS production are increased at the time of fertilization and cell cleavage in bovine zygotes. Hum Reprod 2010;25(11):2762-73.

15. Daponte A, Pournaras S, Zintzaras E, Kallitsaris $A$, Lialios $G$, Maniatis $A N$, et al. The value of a single combined measurement of VEGF, glycodelin, progesterone, PAPP-A, HPL and LIF for differentiating between ectopic and abnormal intrauterine pregnancy. Hum Reprod 2005;20(11):3163-6.

16. Rausch M, Barnhart KT. Serum biomarkers for detecting ectopic pregnancy. Clin Obstet Gynecol 2012;55(2):418-23.

17. Hilali N, Aksoy N, Vural M, Camuzcuoglu $\mathrm{H}$, Taskin A. Oxidative status and serum prolidase activity in tubal ectopic pregnancy. J Pak Med Assoc 2013;63(2):169-72.

18. Shao R, Zou S, Wang X, Feng Y, Brännström $M$, Stener-Victorin $E$, et al. Revealing the hidden mechanisms of smoke-induced fallopian tubal implantation. Biol Reprod 2012;86(4):131.

19. Schlesier K, Harwat M, Böhm V, Bitsch R. Assessment of antioxidant activity by using different in vitro methods. Free Radic Res 2002;36(2):177-87.

20. Janaszewska A, Bartosz G. Assay of total antioxidant capacity: comparison off our methods as applied to human blood plasma. Scand $\mathrm{J}$ Clin Lab Invest 2002;62(3):231-6.

21. Erel O. A novel automated direct measurement method for total antioxidant capacity using a new generation, more stable ABTS radical cation. Clin Biochem 2004;37(4):277-85. 